\title{
Facteurs déterminants le tarif de la vidange mécanique des matières de boues d'assainissement à Dakar
}

\author{
Jean Birane GNING ${ }^{1,2^{*}}$, Cheikh DIOP ${ }^{1}$, Kouassi DONGO ${ }^{3}$ et Doulaye KONÉ ${ }^{4}$ \\ ${ }^{I}$ UniversitéCheikh Anta Diop, avenue Cheikh Anta Diop, Dakar BP 5005 Dakar-Fann, Sénégal. \\ ${ }^{2}$ Swiss Federal Institute of Aquatic Science and Technology (Eawag), Department of Water and Sanitation in \\ Developing Countries (Sandec), Ueberlandstrasse 133, 8600 Duebendorf, Switzerland. \\ ${ }^{3}$ Université Félix Houphouët Boigny (UFHB), 01 BP 1303 Abidjan 01, Côte d'Ivoire. \\ ${ }^{4}$ Bill \&Melinda Gates Foundation, Department of Water, Sanitation \&Hygiene, 500 Fifth Avenue North \\ Seattle, WA 98109 (206) 709-3100. \\ *Auteur correspondant ; E-mail : jeangning@gmail.com; Tél. (+221) 775791302
}

\section{RESUME}

À Dakar, le prix de la vidange mécanique n'est ni fixé ni régulé par l'autorité. Les ménages et les vidangeurs négocient par entente directe des prix jugés élevés. Cette investigation a pour objectif de déterminer les principaux facteurs qui influencent la négociation du prix de la vidange mécanique à Dakar au Sénégal. Une enquête socioéconomique conduite dans la ville de Dakar montre que $88 \%$ des ménages vidangent au moins une fois par an, alors que le choix du mode de vidange est fonction de la disponibilité et de la qualité du service exprimées respectivement par $37 \%$ et $39 \%$ des chefs de ménages. Une observation, des interviews et un audit financier des entreprises de vidange ont servi à la reconstitution des comptes d'exploitation. L'analyse financière a révélé que le coût de la vidange est fonction respectivement de la consommation de carburant, de l'amortissement, de la masse salariale et de la taxe de dépotage. La consommation en carburant représente à elle seule $36 \%$ des charges variables. Ainsi l'établissement de tarifs de vidange mécanique équitable nécessite au préalable le renouvellement des camions vétustes, inefficaces engendrant d'importantes charges de fonctionnement et de maintenance.

(C) 2017 International Formulae Group. All rights reserved.

Mots clés : Assainissement, boues de vidange, vidange mécanique, tarifs.

\section{Driving factors of fecal sludge mechanical emptying tariff in Dakar}

\begin{abstract}
In Dakar, mechanical emptying prices are neither determined nor regulated by authorities. Households and emptiers negotiate by directly agreeing prices, which are considered high. The objective of this study is to determine the main factors influencing the price negotiation of mechanical emptying. A socio-economic survey in Dakar has shown that $88 \%$ of households empty at least once per year, whereas the type of emptying depends on the availability and the quality of services, as expressed by respectively $37 \%$ and $39 \%$ of the heads of households. Observation, interviews and financial audits of emptying companies have been used to reconstitute their accounts. A financial analysis has shown that the cost of emptying depends on fuel consumption, asset depreciation, wages and dumping fees. Fuel consumption alone makes up $36 \%$ of variable
\end{abstract}


costs. Establishing mechanical emptying tariffs would first require replacing the ageing and inefficient trucks, which incur high operation and maintenance costs.

(C) 2017 International Formulae Group. All rights reserved.

Keywords: Sanitation, faecal sludge, mechanical emptying, factors, tariffs.

\section{INTRODUCTION}

En Afrique de l'Ouest plus de $75 \%$ des ménages dans les grandes villes et jusqu'à $100 \%$ dans les petites villes utilisent des installations d'assainissement non collectif, telles que les toilettes publiques, les fosses septiques et les latrines (Agassounon-DjikpoTchibozo et al. 2012; Akiyo et al., 2013 ). Ces installations produisent d'importantes quantités de boues de vidange qui nécessitent un plan de gestion adapté (Still, 2002 ; Kone et al., 2007). Dans plusieurs centres urbains des pays à faible revenu et à revenu intermédiaire, seul moins de la moitié de la production journalière des boues de vidange est collectée et moins de la moitié des boues collectées est traitée (Blackett et al., 2014).

La résolution du problème du déficit d'assainissement nécessite des approches innovantes aussi bien en technologie, qu'en mécanismes de gestion et de détermination des tarifs (Diener et al., 2013; Taweesan et al., 2015). Aujourd'hui, les investigations menées dans la filière des boues de vidange sont plus orientées vers l'innovation technologique et l'organisation; pourtant l'ambiguïté de la tarification de l'assainissement en général et celle des services de vidange en particulier est un frein à l'accès à un assainissement amélioré. En effet, les systèmes traditionnels de tarification des eaux usées indexée à la facture d'eau potable ou à celle de l'électricité ne sont pas adaptés à la filière des boues de vidange. À Dakar, la gestion des boues de vidange est informelle (Chowdhry et Koné, 2012) et le prix de la vidange mécanique n'est ni fixé ni régulé par l'autorité en charge de ce problème. Les ménages et les vidangeurs négocient des prix par entente directe.

Les rares études qui portent sur les tarifs des services de vidange des boues s'orientent vers la volonté et la capacité à payer des ménages (Semiyaga et al., 2015). Cependant, la mise en place de tarifs durables, accessibles aux ménages et qui garantissent la pérennité des entreprises de vidange est sans doute un préalable au développement de la filière des boues de vidange. L'objectif de cette présente investigation est de déterminer les principaux facteurs qui entrent en ligne de compte dans la fixation du prix du service de la vidange mécanique domestique. Il s'est agi d'évaluer (1) le profil socioéconomique des ménages; (2) le profil sanitaire des ménages et (3) les facteurs qui influencent la négociation pour la fixation des prix de la vidange pour le ménage et pour le vidangeur.

L'identification et la levée des contraintes liées à ces facteurs devraient permettre de reconsidérer la filière des boues de vidange et de mettre en place des tarifs au profit du service de la vidange domestique.

\section{MATERIEL ET METHODES \\ Présentation de la zone d'étude}

Cette investigation a été conduite à Dakar; la capitale du Sénégal. La ville de Dakar est située entre les $17^{\circ} 10^{\prime}$ et $17^{\circ} 32^{\prime}$ de longitude Ouest et les $14^{\circ} 53^{\prime}$ et $14^{\circ} 35^{\prime}$ de latitude Nord. Elle s'étend sur $0,28 \%$ du territoire national occupé par $22 \%$ de la population du Sénégal (ANSD, 2014). Dakar est semi-aride avec une saison pluvieuse de juillet à octobre et une saison sèche de novembre à juin (CSE, 2013). La pluviométrie annuelle moyenne est de $514 \mathrm{~mm}$ (WMO, 2016). Dakar dispose d'un assainissement majoritairement non collectif qui produit quotidiennement plus de $5000 \mathrm{~m}^{3}$ de boues (Diop et al., 2014; Sonko et al., 2014). Le marché de la vidange mécanique de la région de Dakar est estimé à 4 millions de dollars (Chowdhry et Koné, 2012) que partagent 208 camions de vidange. Dakar est l'unique ville sénégalaise qui dispose à la fois de trois stations de traitement des boues de vidange (ONAS, 2014). La capacité nominale cumulée des trois stations est de $220 \mathrm{~m}^{3}$ par jour. Pourtant ces infrastructures sont surexploitées avec un dépotage de $1500 \mathrm{~m}^{3}$ par jour (Mbéguéré et al., 2010 ; Dodane et al., 2012). 
Cependant, la filière des boues de vidange de Dakar ne dispose pas de système de tarification homologué comme ceux existant dans les sous-secteurs de l'assainissement collectif et de l'eau potable (WSA, 2012). Les ménages et les vidangeurs marchandent des prix selon des critères et des variables non encore élucidés.

\section{Collecte de données auprès des entreprises de vidange}

La collecte des données est effectuée en deux phases. La première phase a durée quatre mois (entre avril et juillet 2013) pour la collecte des données de base au moyen de groupe de discussion, d'observations participatives et de routage de camions. La deuxième phase a concerné la collecte des données complémentaires au moyen d'entretiens structurés et d'une analyse financière et elle a eu lieu entre janvier et avril de l'année 2014.

\section{Groupes de discussion}

Après l'identification des vidangeurs, ces derniers ont été convoqués avec le soutien des responsables de l'Association des Acteurs de l'Assainissement Autonome du Sénégal (AAAS) pour participer aux discussions de groupes. Les objectifs de l'étude leurs ont été clairement présentés d'une part pour atténuer les biais et d'autre part pour obtenir leur consentement pour la suite de l'investigation. Ensuite, deux groupes ont été constitués: le groupe des propriétaires de camions de vidange et le groupe des chauffeurs de camions de vidange.

Dans le groupe des propriétaires de camions (37 chefs d'entreprises) qui a répondu à l'invitation, les points suivants ont été discutés : les sources d'investissement, le coût de la vidange, la rentabilité de l'activité et les problèmes majeurs rencontrés dans la réalisation de leur activité. Le groupe de discussion avec les vidangeurs a enregistré la participation de 64 chauffeurs de camions de vidange qui ont expliqués la méthode de fixation du prix de la vidange, le mode de paiement des services de vidange, le détail des charges principales de la vidange et les difficultés du sous-secteur.

\section{Observation participative par routage de camion}

Le routage a été effectué avec un camion de vidange de type pompeur d'une capacité de $10 \mathrm{~m}^{3}$ durant quinze jours (du 2 au 17 mars 2014). Le choix du camion de ce type est motivé par le fait que ce dernier est le plus couramment sollicité par les ménages de Dakar.

Durant cette période, le travail a consisté à intégrer l'équipage d'un camion et s'impliquer pleinement dans l'activité de vidange. Les informations suivantes ont été recueillies : les zones de fréquentes vidanges ; la nature des ouvrages; la distance de pompage ; la durée du transport; le temps de vidange de la fosse, le temps de dépotage, le temps mort, la distance parcourue; la consommation en carburant du camion et de la pompe d'aspiration par rotation, le prix de la vidange d'une fosse, le mode de paiement, la taxe de dépotage, la fréquence des tracasseries policières.

\section{Interviews avec les chefs d'entreprises de vidange}

Le guide d'entretien utilisé pour cette interview est de type structuré. Il est composé de quatre questions portant sur (1) le profil des entreprises; (2) les charges et les recettes de la vidange; (3) le seuil de rentabilité des entreprises en termes de nombre de voyages et (4) les facteurs déterminants la fixation des prix.

La répartition des entreprises en fonction du nombre de camions (Figure 2) a permis de d'identifier les différentes tailles d'entreprises existante dans la ville de Dakar. Sur cette base, les entreprises sont réparties en trois catégories: Les petites entreprises disposant d'un camion, les entreprises moyennes ayant deux camions et les grandes sont celles disposant d'au moins 3 camions.

Dans chaque catégorie, les deux premiers chefs d'entreprises de vidange volontaires ont été interviewés pour compléter 
les données comptables financières spécifiques obtenues directement.

\section{Enquête auprès des ménages}

Pour prendre en compte l'hétérogénéité des installations d'assainissement à Dakar, marquée par l'existence d'un réseau d'égout long de $1326 \mathrm{~km}$ d'une petite expérience en réseau semi-collectif et d'une majorité d'ouvrages individuels, une méthode de stratification à trois niveaux est conduite comme le préconisent Chowdhry et Kone, (2012). Ainsi, les quartiers sont classés en sous-groupes homogènes. L'homogénéité est définie en fonction du niveau d'urbanisation et du type d'ouvrage d'assainissement. Les quartiers qui présentent les mêmes caractéristiques sont considérés comme homogènes et classés dans un même groupe colorié à la même couleur sur la carte de la ville (Figure 1). La sélection des quartiers à enquêter dans chaque zone homogène est aléatoire tandis que la détermination de l'échantillon des ménages à enquêter est un choix raisonné proportionnellement à la densité de la population du quartier.

Dans les habitudes sénégalaises, plusieurs ménages peuvent partager le même ouvrage d'assainissement individuel, comme c'est le cas des maisons à étages, l'enquête ne prend en compte qu'un ménage par concession. Ainsi, après le regroupement des quartiers en zone homogène, la population étudiée est celle bénéficiant effectivement d'un assainissement non collectif. À Dakar la population cible bénéficiaire de l'assainissement non collectif est estimée à 2 574065 personnes réparties dans 279790 ménages (ANSD, 2014).

Compte tenu de la complexité de l'enquête ménage, du coût onéreux et de la limite du budget, la méthodologie d'échantillonnage par quotas est adoptée. Ainsi, un quota d'une taille de 502 chefs de ménages desservis par l'assainissement non collectif a été enquêté sur la base de leur consentement.

Le questionnaire était conçu pour obtenir des informations sur le prix et la volonté à payer pour le service de vidange mécanique. Il comportait quatre sections. L'introduction ou l'explication du but de l'étude est obligatoirement lue pour demander l'approbation du chef de ménages. Les chefs de ménages consentants sont enquêtés dans l'anonymat. La section A renseigne sur le profil socio-économique (l'âge, le sexe, le niveau d'éducation, la taille du ménage, le revenu, les équipements disponibles pour le ménage).

La section B porte sur l'assainissement en général (gestion des ordures ménagères et des eaux usées). La section $\mathrm{C}$ porte spécifiquement sur le service de vidange mécanique. Les principaux paramètres abordés dans cette section sont: le coût de la vidange mécanique, le mode de paiement et le consentement à payer (CAP) des ménages pour une amélioration du service. Le consentement à payer est obtenu sur une base d'enchère entre l'enquêteur et le chef de ménages jusqu'au tarif maximum que ce dernier est prêt à payer (EDE/ICEA, 2009).

Avant l'enquête, un échantillon aléatoire de 10 ménages a été étudié au préalable pour le pré-test du questionnaire. Cela a permis d'évaluer l'ambigüité de certaines questions et d'améliorer la qualité du questionnaire (Ngoutane Pare et al., 2012). L'enquête a été effectuée entre avril et mai 2013. Le choix du ménage à interviewer était systémique avec un premier chef de ménage sélectionné au hasard. Les autres ménages suivants sont sélectionnés en comptant 10 maisons à partir de celle précédemment enquêtée. Cette sélection est faite par l'enquêteur. Si un domicile comporte plusieurs ménages, l'enquêteur se déplace dans une direction différente. Dans la première maison présentant ce cas de figure, l'enquêteur doit sélectionner le ménage vivant dans la partie droite. Dans la maison suivante qui a la même situation, il n'enquête que le ménage d'en face. Si la situation se présente de nouveau, c'est le ménage de la gauche qui est enquêté et dans la maison suivante, il n'enquête que le ménage à sa droite.

\section{Analyse des données \\ Analyse des données de terrain}

Les données ont été analysées à l'aide des logiciels Windows Excel et SPSS 15.0 (SPSS, 2006). Le test $\mathrm{t}$ de Student apparié a été utilisé avec un seuil de confiance de 0,05. Des statistiques descriptives, telles que la fréquence et le pourcentage, ont été utilisées 
pour établir les informations générales. Par la suite un plan de tabulation a été élaboré à partir des bases de données pour réaliser les graphiques et les analyses. Pour pallier le biais observé sur les données en ce qui concerne le niveau de revenu des ménages, l'Analyse à Composante Principale (ACP) a été adoptée (Mbeguere et al., 2011).

L'adaptation de la méthodologie de calcul de l'Indice de Richesse (IR) développée par Filmer et Pritchett (2001) a permis de construire l'IR des ménages. Cette approche a également été utilisée dans l'étude de la volonté à payer pour les services d'eau potable et d'assainissement au Sénégal (EDE/ICEA, 2009). L'IR est évalué sur la base du patrimoine du ménages tels que : les caractéristiques de l'habitat, les biens qu'ils possèdent et le statut d'occupation du logement. Ces éléments sont peu manipulables par les enquêtés et par conséquent ils sont plus fiables qu'une question sur le niveau de revenu (EDE/ICEA, 2009).

La construction de l'IR est faite en quatre étapes: pour le choix des variables, dix variables ont été retenus et réparties en trois groupes: (1) les équipements du ménage (vélo, moto, voiture, radio, téléviseur, réfrigérateur); (2) les caractéristiques de l'habitat (branchement privé d'eau potable, électricité, téléphone) et (3) le statut d'occupation de l'habitat (propriétaire ou locataire).

Pour la détermination de l'ACP, chaque bien est défini en fonction d'un poids factoriel qui correspond au score factoriel du bien. Selon Mbeguere et al. (2011) le score est calculé selon la formule suivante:

$\mathrm{S}_{(\mathrm{i}, \mathrm{i})}=\left(\mathrm{V}_{\mathrm{i}}-\mathrm{M}_{\mathrm{i}}\right) \times \mathrm{F}_{\mathrm{i}} / \mathrm{E}_{\mathrm{i}}$

Avec $S(i, j)$ : le score du ménage i pour le bien $\mathrm{j} ; \mathrm{V}_{\mathrm{i}}$ : la valeur du bien $\mathrm{j} ; \mathrm{M}_{\mathrm{i}}$ : la moyenne $j ; F_{j}$ : le scort factoriel jet $E_{j}:$ Ecart type $\mathrm{j}$

La moyenne $\mathrm{j}$ et l'écart-type $\mathrm{j}$ sont calculés sur la population $\mathrm{S}$ de l'échantillon. Le score factoriel $\mathrm{j}$ est la valeur estimée du facteur pour le bien $\mathrm{j}$. Valeur $\mathrm{j}=0$ si le ménage ne possède pas le bien $\mathrm{j}$; Valeur $\mathrm{j}=1$ si le ménage possède le bien $\mathrm{j}$.

Le calcul de la note de l'indice de richesse de chaque ménage : elle est obtenue par la sommation des scores obtenus pour les différents biens. Elle constitue l'indice de richesse.

La répartition en quintiles: elle correspond à la division de l'échantillon en cinq groupes de même taille. Dans cette étude l'échantillon est divisé en quintiles selon le niveau de richesse des ménages: $I_{1}$ (ménages les plus riches), $\mathrm{IR}_{2}$ (ménages riches), $\mathrm{IR}_{3}$ (ménages moyens), $\mathrm{IR}_{4}$ (ménages pauvres) et $\mathrm{IR}_{5}$ (ménages les plus pauvres).

\section{Analyse des données financières}

L'analyse des données financières a consisté à la reconstitution des comptes d'exploitation et des flux financiers à l'évaluation du seuil de rentabilité et des prix d'équilibre de chaque entreprise. Cette analyse s'est appuyée sur la comptabilité analytique et plus particulièrement sur la technique de l'audit.

Suivant le type de données financières collectées, plusieurs approches sont adoptées pour la validation des données et la gestion des incertitudes. Pour l'analyse des données de cette investigation la multiplication comparative des sources a été utilisée. Dans la mesure du possible et au cas par cas, chaque donnée non disponible dans la comptabilité a été estimée par le croisement d'informations, la comparaison avec les données des entreprises formelles. Le seuil de rentabilité est calculé suivant l'équation suivante (Batsch, 2003).

$\mathrm{S}_{\mathrm{r}}=\mathrm{C}_{\mathrm{f}} / \mathrm{TMC}_{\mathrm{v}}=\mathrm{CA} \times \mathrm{C}_{\mathrm{f}} / \mathrm{MC}_{\mathrm{V}}$

$\mathrm{S}_{\mathrm{r}}$ : Seuil de rentabilité ; $\mathrm{TMC}_{\mathrm{v}}$ : Taux de Marge sur Coûts variables; $\mathrm{C}_{\mathrm{f}}$ : Coûts fixes ; $\mathrm{MC}_{\mathrm{v}}$ : Marge sur les Coûts variables; CA: chiffre d'affaires

La marge sur les couts variable est obtenue suivant la formule suivante :

$\mathrm{MC}_{\mathrm{v}}=\mathrm{CA}-\mathrm{C}_{\mathrm{v}}$

$\mathrm{MC}_{\mathrm{v}}$ Marge sur les Coûts variables; CA : Chiffre d'Affaires ; $\mathrm{C}_{\mathrm{v}}$ : Coûts variables

Le seuil de rentabilité en valeur est atteint quand:

$\left(\mathrm{MC}_{\mathrm{V}}\right.$ unitaire $\left.\times \mathrm{Q}\right) / \mathrm{Cf} \geq 1$

$\mathrm{MC}_{\mathrm{v}}$ Marge sur les Coûts variables; $\mathrm{C}_{\mathrm{f}}$ : Coûts fixes; $\mathrm{Q}$ : nombre de rotations/vidanges; $\mathrm{C}_{\mathrm{f}}$ : Coûts fixes.

Le seuil de rentabilité est principalement calculé en monnaie mais, pour des besoins de précision, il est par moment évalué en termes de nombre de voyages. 


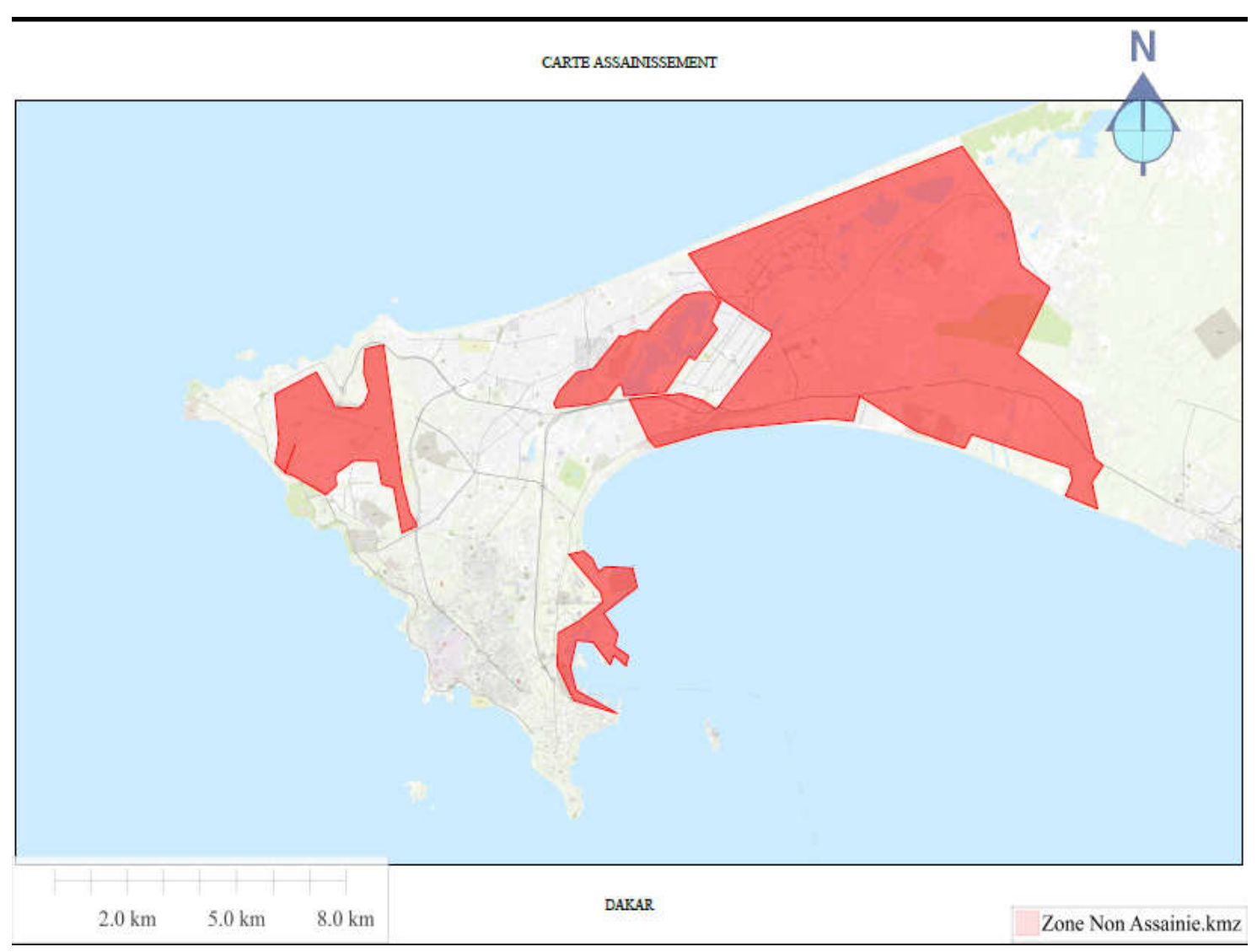

Figure 1 : Carte de Dakar avec les zones d'assainissement non collectif.

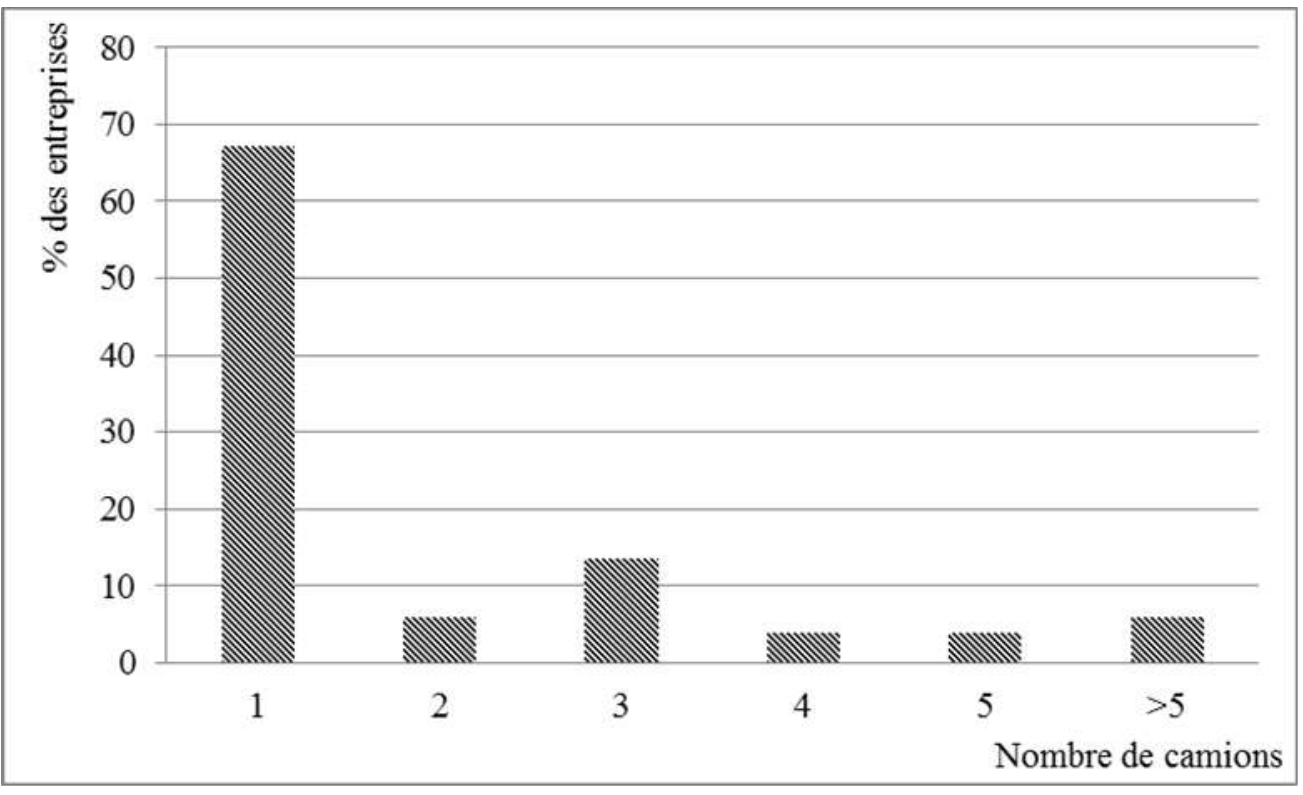

Figure 2 : Répartition des entreprises en fonction du nombre de camions. 


\section{RESULTATS}

\section{Profil socioéconomique des ménages}

Les caractéristiques socio-

économiques des ménages étudiés portent principalement sur le sexe, le niveau d'instruction, l'indice de richesse, la taille des ménages, et le statut d'occupation des habitations.

Les résultats de l'étude montrent que la majorité des enquêtés sont des femmes. En effet, $63 \%$ des chefs de ménages sont des femmes contre $37 \%$ qui sont des hommes.

Le taux d'instruction des chefs de ménages quant à lui, atteint $77 \%$ et les enquêtés qui ont le niveau moyen-secondaire représentent 51\% de l'échantillon (Tableau 1). Par ailleurs 23\% des chefs de ménages n'ont jamais été scolarisé.

La classification des chefs de ménage selon l'indice de richesse distingue cinq quintiles de classements des ménages en fonction de leurs niveaux de richesse: les ménages les plus riches $\left(\mathrm{IR}_{1}\right)$; les ménages riches $\left(\mathrm{IR}_{2}\right)$ les ménages moyennement riche $\left(\mathrm{IR}_{3}\right)$, les ménages pauvres $\left(\mathrm{IR}_{4}\right)$ et les ménages les plus pauvres $\left(\mathrm{IR}_{5}\right)$. Chaque quintile d'IR représente une proportion de $20 \%$ des ménages enquêtés.

En ce qui concerne la taille des ménages, les résultats du sondage montrent qu'elle varie entre 1 et 30 personnes avec une moyenne de 9 personnes par ménage (Tableau 2). Il faut cependant noter qu'au cours de l'enquête $16,5 \%$ des interviewés soit 83 chefs de ménages ont refusé de répondre à cette question.

Durant l'enquête deux statuts ont été distingués par rapport à l'occupation de l'habitat: le chef de ménage est soit propriétaire, soit simple locataire. Au total, plus de la moitié soit $68 \%$ des chefs de ménages déclarent être propriétaires de leurs habitations contre $32 \%$ qui sont des locataires (Tableau 3). Ce taux est plus important chez les ménages moyennement riches dont $90 \%$ habitent leurs propres maisons. Même pour les ménages les plus pauvres, $58 \%$ sont propriétaires de leurs domiciles.

\section{Profil sanitaire des ménages}

Les principaux modes d'approvisionnement en eau potable rencontrés dans la ville de Dakar sont: les branchements privés, les bornes fontaines, les forages, les puits et les revendeurs privés (Tableau 4). L'enquête auprès des ménages montre une prédominance à $82 \%$ des branchements privés comme principal mode de fourniture en eau potable. L'approvisionnement en eau potable à partir des bornes fontaines arrive en deuxième position avec $8 \%$ des ménages alors que moins de $1 \%$ des ménages s'approvisionne à partir des puits.

Les résultats révèlent également que $99 \%$ des ménages enquêtés disposent d'ouvrage d'assainissement à domicile et $1 \%$ continue de chercher un lieu d'aisance. Les types d'ouvrages individuels les plus utilisés à Dakar sont les fosses septiques qui représentent $82 \%$, suivies des toilettes à chasse manuelle (7\%). Tous les types d'ouvrages individuels rencontrés dans les ménages et consignés dans le Tableau 5 produisent d'importantes quantités de boues évaluées à $5000 \mathrm{~m}^{3}$.

Sur l'ensemble des chefs de ménages enquêtés, seul $40 \%$ des répondants vidangent automatiquement après le remplissage de la fosse (Tableau 6). En effet, $9 \%$ des chefs de ménage ne vidange seulement que quand ils ont de l'argent et les $29 \%$ déclarent n'avoir jamais effectué une vidange de leurs fosses.

Pour ce qui est du mode de vidange, la vidange manuelle est pratiquée par $47 \%$ des ménages enquêtés contre $42,5 \%$ seulement pour la vidange mécanique. Pour avoir une meilleure vidange mais plus couteuse, 9,3\% des ménages combinent les deux modes de vidange. La mode de vidange est aussi corrélée à l'indice de richesse des ménages. À Dakar la répartition en fonction de l'indice de richesse montre que les ménages les plus riches $\left(\mathrm{IR}_{1}\right)$ pratiquent la vidange mécanique pour près de $70 \%$ alors que $54 \%$ des ménages les plus pauvres $\left(\mathrm{IR}_{5}\right)$ font appel aux vidangeurs manuels. 


\section{Consentement à payer pour une opération de vidange mécanique}

Les résultats de l'enquête révèlent un consentement à payer inférieur au prix moyen de la vidange mécanique qui s'élève actuellement à un montant de 25000 Francs CFA (Tableau 7 et Tableau 9). La volonté à payer pour la vidange mécanique varie dans le même sens que: l'indice de richesse des ménages, la qualité du service et la fréquence de vidange. Plus de $51 \%$ des ménages les plus riches $\left(\mathrm{IR}_{1}\right)$ et les riches $\left(\mathrm{IR}_{2}\right)$ sont prêts à payer un montant compris entre 20000 FCFA et 25000 FCFA alors que seulement $15 \%$ des ménages les plus pauvres $\left(\mathrm{IR}_{5}\right)$ acceptent de payer ce même prix. Les ménages les plus riches $\left(\mathrm{RI}_{1}\right)$ ont une volonté à payer pour un montant supérieure à $10 \quad 000 \quad$ FCFA contrairement aux plus pauvres $\left(\mathrm{IR}_{5}\right)$ qui réclament parfois la gratuité du service. Il existe cependant des exceptions ou les ménages pauvres acceptent de payer plus que les riches à cause de l'importance de la taille de leurs ménages qui engendre une fréquence de vidange plus élevée. En effet, les résultats de l'étude révèlent que pour $74 \%$ des ménages de Dakar, la vidange est pratiquée au moins une fois par an.

La disponibilité et la qualité du service déterminent le choix de la vidange mécanique pour $76 \%$ des chefs de ménages de Dakar (Tableau 8). Même si le niveau du coût de la vidange mécanique demeure problématique pour les ménages, il n'est pas une variable fondamentale. C'est plutôt, la fréquence de vidange élevée et le faible rendement des pompes des camions de vidange qui ont motivés $39 \%$ des chefs de ménages à considérer la qualité du service comme le premier facteur de choix du mode de vidange. Pour $37 \%$ des chefs de ménage, c'est plutôt la disponibilité du service qui est le critère de choix de la vidange mécanique. Dans certains quartiers éloignés des stations de traitement des boues de vidange ou en zones d'habitats spontanés, il est parfois difficile de trouver un vidangeur mécanique du fait de l'inaccessibilité.
Le critère coût de la vidange mécanique arrive bien après la qualité et la disponibilité du service. C'est seulement $16 \%$ des chefs de ménages qui fondent le choix du mode de vidange mécanique sur le niveau du prix. La classification des critères de choix du mode de vidange varie également en fonction de l'indice de richesse. En effet, plus le ménage est riche, moins le prix de la vidange est déterminant dans le choix du mode de vidange.

\section{Mode de paiement de la vidange mécanique}

Selon $90 \%$ des chefs de ménages enquêtés, le paiement de la vidange et du transport des boues se fait en intégralité et immédiatement après l'exécution du service. Mais, il existe un début de paiement différé pratiqué par $10 \%$ des ménages enquêtés.

\section{Déterminants du coût théorique de la vidange chez l'opérateur privé}

L'accessibilité de la concession ou de l'ouvrage à vidanger, la distance par rapport au site de dépotage, le volume de la fosse à vidanger ou la taille du camion sont autant d'éléments évoqués dans le groupe de discussion comme étant des facteurs qui influencent le coût de la vidange mécanique. Les facteurs financiers liés à la rentabilité des entreprises qui concourent également à la variation des coûts. Les données primaires de l'activité de la vidange mécanique des boues à Dakar présentées dans le Tableau 12 révèlent que l'âge des camions varie entre 20 et 45 ans. Ainsi, la consommation moyenne de carburant par voyage de moins de $20 \mathrm{~km}$ s'élève à un montant de 9000 Francs CFA soit 15 litres de carburant.

Les entreprises de vidange ont généralement deux sources de revenus: les recettes principales issues de l'activité de vidange des boues domestiques et les recettes secondaires provenant des autres services de vidanges non domestiques tels que : l'entretien du réseau d'égout, le curage des caniveaux d'eau pluviales, le nettoiement des points d'eau, le curage des eaux usées et des boues industrielles. 
Les charges d'exploitation des entreprises de vidange étudiées sont constituées de charges fixes et de charges variables. Les charges fixes sont composées : des salaires; du forfait pour le matériel de travail; des fournitures de bureau; des factures d'électricité et d'eau; de la visite technique des véhicules; de l'assurance; de la location d'un bureau et d'un parking; du forfait des frais d'entretien et de la maintenance et des dotations aux amortissements. Les charges fixes varient en fonction de la taille de l'entreprise de vidange. Les charges variables sont composées de l'achat de carburant, de la taxe de dépotage, de la facture de téléphone, des frais de réparation des pannes des camions et des primes.

\section{Compte d'exploitation et rentabilité}

La reconstitution des comptes d'exploitation prévisionnels après cinq ans d'exercice fournissent des marges qui varient entre un minimum de $9 \%$ pour les petites entreprises et un maximum de $53 \%$ pour les grandes entreprises. Ces résultats sont une illustration de la rentabilité de l'activité de la vidange mécanique. Les comptes d'exploitation des entreprises de différentes tailles étudiées sont représentés dans les Tableaux 10,11 et 12 .

Les petites entreprises dégagent un résultat après impôts de l'ordre de $2 \%$ du chiffre d'affaire. Cette faible marge s'explique par l'importance des charges (Tableau 10). Les vidangeurs mécaniques ont une masse salariale qui représente $14 \%$ du chiffre d'affaires. Les petites entreprises de vidange sont généralement informelles. Cette taille d'entreprise se distingue également par une charge d'entretien et de maintenance des camions de l'ordre de $5 \%$ du chiffre d'affaires. Les charges variables représentent $56 \%$ du chiffre d'affaire. La consommation en carburant représente à elle seule $36 \%$ des charges variables soit $20 \%$ du chiffre d'affaire. Le carburant tableau en tête, car c'est une charge motrice de l'activité. La vétusté des camions et des pompes favorisent en partie l'augmentation de la consommation de carburant. La taxe de dépotage représente $12 \%$ des recettes et la somme des autres charges variables ne dépasse pas 5\% (Tableau 10)

Les résultats de l'analyse financière révèlent que les entreprises moyennes réalisent des chiffres d'affaires moyens évalués à un montant de 46800000 FCFA (Tableau 11). Les différentes charges évoluent dans le même sens que celles des petites entreprises avec le carburant en tête suivie des salaires, de la dotation aux amortissements et de la taxe de dépotage. Le solde cumulé après cinq années d'activité atteint $39 \%$ du chiffre d'affaires. Le seuil d'équilibre est donc atteint avec seulement 39013377 FCFA de chiffre d'affaires correspondant à 260 jours d'activité. Ainsi, dans les conditions actuelles d'activité des entreprises qui disposent de 2 camions de vidange, le coût d'équilibre est de 20840 FCFA par voyage. A partir de ce tarif, l'ensemble des charges est égal aux recettes. Une comparaison de ce coût d'équilibre avec le prix moyen actuellement payé par les ménages pour une vidange permet de constater que les entreprises moyennes font un profit d'au moins $16,6 \%$.

Les grandes entreprises possèdent au moins trois (3) camions et parviennent à réaliser plus facilement des économies d'échelle. Le compte d'exploitation de ces entreprises, montre un résultat positif qui représente $10 \%$ de leur chiffre d'affaires. À l'exception des dotations aux amortissements, les autres charges évoluent dans les mêmes proportions que celles des petites et des moyennes entreprises. Les prévisions du compte d'exploitation sur une durée de cinq (5) années montrent un solde de l'ordre de $49 \% \mathrm{du}$ chiffre d'affaires (Tableau 12). L'évaluation du seuil d'équilibre des grandes entreprises illustre l'avantage de l'économie d'échelle. En effet, plus la taille de l'entreprise de vidange est grande, plus le prix d'équilibre est faible, car les charges fixes évoluent faiblement. Le seuil d'équilibre est atteint avec un chiffre d'affaires d'un montant de 53758972 FCFA correspondant à 239 jours d'activité. Ainsi, le coût d'équilibre est d'un montant de 19145 FCFA par voyage soit une marge par voyage de l'ordre de $23 \%$ du prix moyen actuellement payé pour la vidange. 
Tableau 1: Répartition des chefs de ménages selon le niveau d'instruction.

\begin{tabular}{|c|c|}
\hline Niveau d'instruction & Pourcentage \\
\hline Non scolarisé & $23 \%$ \\
\hline Primaire & $26 \%$ \\
\hline Moyen-Secondaire & $33 \%$ \\
\hline Supérieur & $18 \%$ \\
\hline Total & $100 \%$ \\
\hline
\end{tabular}

Tableau 2: Répartition des ménages en fonction de la taille.

\begin{tabular}{lrrrrrrrr} 
Intervalles nombre de Personnes & {$[1-5]$} & {$[6-10]$} & {$[11-15]$} & {$[16-20]$} & {$[21-25]$} & {$[26-30]$} & Total \\
\hline & & & & & & & & \\
\% des ménages & 20,5 & 45,6 & 26,5 & 6,2 & 0,7 & 0,5 & $\mathbf{1 0 0 , 0}$ \\
\hline
\end{tabular}

Tableau 3: Statut d'occupation de la maison.

\begin{tabular}{rlll}
\hline Statut & & Propriétaire & Locataire \\
IR1 & $58 \%$ & $42 \%$ & \\
IR2 & $54 \%$ & $46 \%$ \\
IR3 & $90 \%$ & $10 \%$ \\
IR4 & $73 \%$ & $27 \%$ \\
IR5 & $58 \%$ & $42 \%$ \\
Total & $\mathbf{6 8 \%}$ & $\mathbf{3 2 \%}$ \\
\hline
\end{tabular}

$\mathrm{IR}_{1}$ : les ménages les plus riches; $\mathrm{IR}_{2}$ : les ménages riches ; $\mathrm{IR}_{3}$ : les ménages moyennement riches ; $\mathrm{IR}_{4}$ : les ménages pauvres ; $\mathrm{IR}_{5}$ : les ménages les plus pauvres

Tableau 4: Mode/source d'approvisionnement en eau.

\begin{tabular}{lcccccc}
\hline Source & Branchement privé & Borne fontaine & Vendeurs d'eau & puits & Autre & Total \\
\hline Effectif & 411 & 40 & 39 & 2 & 10 & $\mathbf{5 0 2}$ \\
Pourcentage & $82 \%$ & $8 \%$ & $8 \%$ & $0,4 \%$ & $2 \%$ & $\mathbf{1 0 0 \%}$ \\
\hline
\end{tabular}

Tableau 5: La typologie des toilettes utilisées par les ménages.

\begin{tabular}{|c|c|c|c|c|c|c|c|}
\hline Technologies & $\begin{array}{r}\text { réseau semi- } \\
\text { collectif }\end{array}$ & $\begin{array}{r}\text { Fosse } \\
\text { septique }\end{array}$ & $\begin{array}{r}\text { Latrine } \\
\text { traditionnelle }\end{array}$ & TCM & VIP & Autre & Total \\
\hline Dakar & $5,0 \%$ & $82 \%$ & $1,8 \%$ & $7 \%$ & $3,8 \%$ & $0,4 \%$ & $100,0 \%$ \\
\hline
\end{tabular}


Tableau 6: Mode de gestion des ouvrages non collectifs pleins.

\begin{tabular}{lrr}
\hline Désignation & Effectif & Pourcentage \\
\hline Vidanger automatiquement & 203 & $40 \%$ \\
Vidanger quand j'ai de l'argent & 45 & $9 \%$ \\
Fermer la fosse & 3 & $1 \%$ \\
Ouvrage jamais remplie & 145 & $29 \%$ \\
Contacter l'opérateur et attendre & 14 & $3 \%$ \\
Sans réponse & 92 & $18 \%$ \\
Total & $\mathbf{5 0 2}$ & $\mathbf{1 0 0 \%}$ \\
\hline
\end{tabular}

Tableau 7: Consentement à payer pour le service de de vidange en Francs CFA.

\begin{tabular}{lrrrrrrrr}
\hline Mon & {$[5001-10$} & {$[10001-$} & {$[15001-$} & {$[20001-$} & {$[25001-$} & {$[30001-35$} & {$[35001-$} & Totale \\
& $000]$ & $15000]$ & $20000]$ & $25000]$ & $30000]$ & $000]$ & $40000]$ & \\
\hline IR1 & $0 \%$ & $6 \%$ & $19 \%$ & $52 \%$ & $23 \%$ & $0 \%$ & $0 \%$ & $100 \%$ \\
IR2 & $11 \%$ & $6 \%$ & $22 \%$ & $50 \%$ & $11 \%$ & $0 \%$ & $0 \%$ & $100 \%$ \\
IR3 & $4 \%$ & $4 \%$ & $42 \%$ & $35 \%$ & $4 \%$ & $12 \%$ & $0 \%$ & $100 \%$ \\
IR4 & $6 \%$ & $25 \%$ & $25 \%$ & $19 \%$ & $25 \%$ & $0 \%$ & $0 \%$ & $100 \%$ \\
IR5 & $11 \%$ & $26 \%$ & $32 \%$ & $16 \%$ & $11 \%$ & $0 \%$ & $5 \%$ & $100 \%$ \\
Total & $14 \%$ & $25 \%$ & $19 \%$ & $15 \%$ & $13 \%$ & $11 \%$ & $2 \%$ & $100 \%$ \\
\hline
\end{tabular}

$\mathrm{IR}_{1}$ : les ménages les plus riches; $\mathrm{IR}_{2}$ : les ménages riches ; $\mathrm{IR}_{3}$ : les ménages moyennement riches ; $\mathrm{IR}_{4}$ : les ménages pauvres ; $\mathrm{IR}_{5}$ : les ménages les plus pauvres

Tableau 8: Critères de choix du mode de vidange mécanique.

\begin{tabular}{lr}
\hline Critères & Pourcentage \\
\hline Prix & $16 \%$ \\
disponibilité & $37 \%$ \\
qualité service & $39 \%$ \\
autres & $8 \%$ \\
Total & $100 \%$ \\
\hline
\end{tabular}

Tableau 9: Données de base de l'activité des entreprises de vidange.

\begin{tabular}{lc}
\hline Désignation & Ratios \\
\cline { 2 - 2 } Intervalle d'âge des camions en années & 20 à 45 \\
Nombre moyen de jours de travail par an & 312 \\
Nombre moyen de voyages par camion et par jour & 3 \\
Consommation en carburant par voyage en FCFA & 9000 \\
Taxe de dépotage $1 \mathrm{~m}^{3}$ de boues en FCFA & 300 \\
Taille moyenne des camions de vidange en $\mathrm{m}^{3}$ & 8 à 10 \\
Prix moyen d'une vidange en FCFA & 25000
\end{tabular}


Tableau 10: Compte d'exploitation des petites entreprises.

\begin{tabular}{|c|c|c|}
\hline Désignation & En \% & Montants en FCFA \\
\hline Chiffre d'affaires & $100 \%$ & 23400000 \\
\hline Charges variables & $63 \%$ & 14640000 \\
\hline Achat de carburant & $36 \%$ & 8424000 \\
\hline Taxe de dépotage & $12 \%$ & 2808000 \\
\hline Téléphone & $3 \%$ & 624000 \\
\hline Réparation & $3 \%$ & 600000 \\
\hline Prime au personnel & $5 \%$ & 1248000 \\
\hline Autres charges & $4 \%$ & 936000 \\
\hline Charges fixes & $35 \%$ & 8210600 \\
\hline Salaire personnel & $14 \%$ & 3336000 \\
\hline Chaussures de sécurité & $0 \%$ & 45000 \\
\hline gants & $0 \%$ & 78000 \\
\hline Masques (cache nez) & $0 \%$ & 93600 \\
\hline balaie & $0 \%$ & 3000 \\
\hline Seau & $0 \%$ & 2000 \\
\hline Fournitures de bureau & $0 \%$ & 50000 \\
\hline visite technique & $0 \%$ & 20000 \\
\hline Assurance & $1 \%$ & 152000 \\
\hline Entretien et maintenance & $5 \%$ & 1200000 \\
\hline Dotation aux amortissements & $14 \%$ & 3231000 \\
\hline Charges totales & $98 \%$ & 22850600 \\
\hline Résultat Avant Impôt & $2 \%$ & 549400 \\
\hline Impôts sur les sociétés & $1 \%$ & 137350 \\
\hline Résultat net après Impôt & $2 \%$ & 412050 \\
\hline Solde sur 5 ans & $9 \%$ & 2060250 \\
\hline
\end{tabular}


Tableau 11: Compte d'exploitation des entreprises moyennes.

\begin{tabular}{|c|c|c|}
\hline Désignation & En \% & Montants en FCFA \\
\hline Total Chiffre d'affaires & $100 \%$ & 46800000 \\
\hline Charges variables & $61 \%$ & 28608000 \\
\hline Achat de carburant & $36 \%$ & 16848000 \\
\hline Taxe de dépotage & $12 \%$ & 5616000 \\
\hline Téléphone & $2 \%$ & 936000 \\
\hline Réparation & $2 \%$ & 840000 \\
\hline Prime au personnel & $5 \%$ & 2496000 \\
\hline Autres charges (police, association etc.) & $4 \%$ & 1872000 \\
\hline Charges fixes & $28 \%$ & 13293200 \\
\hline Salaire personnel & $14 \%$ & 6672000 \\
\hline Chaussures de sécurité & $0 \%$ & 90000 \\
\hline gants & $0 \%$ & 156000 \\
\hline Masques (cache nez) & $0 \%$ & 187200 \\
\hline Balaye & $0 \%$ & 6000 \\
\hline Seau & $0 \%$ & 4000 \\
\hline Fournitures de bureau & $0 \%$ & 50000 \\
\hline Visite technique & $0 \%$ & 40000 \\
\hline Assurance & $1 \%$ & 304000 \\
\hline Entretien et maintenance & $3 \%$ & 1200000 \\
\hline Dotation amortissements & $14 \%$ & 6456000 \\
\hline Charges totales & $90 \%$ & 41901200 \\
\hline Résultat Avant Impôt & $10 \%$ & 4898800 \\
\hline Impôts sur les sociétés & $3 \%$ & 1224700 \\
\hline Résultat après Impôt & $8 \%$ & 3674100 \\
\hline Solde sur 5 ans & $39 \%$ & 18370500 \\
\hline
\end{tabular}


Tableau 12: Compte d'exploitation des grandes entreprises.

\begin{tabular}{|c|c|c|}
\hline Désignation & En \% & Montants en FCFA \\
\hline Total Chiffre d'affaires & $100 \%$ & 70200000 \\
\hline Charges variables & $61 \%$ & 42708000 \\
\hline Achat de carburant & $36 \%$ & 25272000 \\
\hline Taxe de dépotage & $12 \%$ & 8424000 \\
\hline Téléphone & $2 \%$ & 1560000 \\
\hline Réparation & $1 \%$ & 900000 \\
\hline Prime au personnel & $5 \%$ & 3744000 \\
\hline Autres charge (police, association etc.) & $4 \%$ & 2808000 \\
\hline Charges fixes & $26 \%$ & 18245300 \\
\hline Salaire personnel & $10 \%$ & 7200000 \\
\hline Chaussures de sécurité & $0 \%$ & 135000 \\
\hline Gants & $0 \%$ & 234000 \\
\hline Masques (cache nez) & $0 \%$ & 280800 \\
\hline balaie & $0 \%$ & 4500 \\
\hline Seau & $0 \%$ & 6000 \\
\hline Fournitures de bureau & $0 \%$ & 50000 \\
\hline visite technique & $0 \%$ & 60000 \\
\hline Assurance & $1 \%$ & 456000 \\
\hline Entretien et maintenance & $0 \%$ & 150000 \\
\hline Dotation aux amortissements & $14 \%$ & 9669000 \\
\hline Charges totales & $87 \%$ & 60953300 \\
\hline Résultat Avant Impôt & $13 \%$ & 9246700 \\
\hline Impôts sur les sociétés & $3 \%$ & 2311675 \\
\hline Résultat après Impôt & $10 \%$ & 6935025 \\
\hline Solde sur 5 ans & $49 \%$ & 34675125 \\
\hline
\end{tabular}




\section{DISCUSSION}

La situation des femmes chefs de ménages majoritairement observée par cette investigation est en contradiction avec les coutumes en Afrique subsaharienne où les ménages dirigés par des femmes demeurent relativement rares (Pilon et Vignikin, 2007). Toutefois ce résultat est un atout car, au Sénégal, les femmes sont au premier plan pour l'entretien des toilettes et l'hygiène domestique.

L'important taux d'instruction observé est tout aussi un avantage dans la mesure où il peut engendrer une meilleure prise en charge de l'hygiène dans les ménages et par conséquent favoriser la collecte des boues domestiques. En effet, selon le rapport de SWASA (2013) les chefs de ménages instruits ont une meilleure compréhension des risques liés à une mauvaise gestion des boues de vidange.

Pour ce qui est de la taille moyenne des ménages, elle est bien au-dessus de celle des derniers recensements dans la ville. En effet, selon ANSD (2014) la taille moyenne des ménages dakarois serait de 6 personnes. Cet écart peut s'expliquer par le fait que cette étude porte sur les zones non raccordées aux réseaux d'égout alors que ces dernières sont en général les plus densément peuplées. Toutefois, vu que la taille des ouvrages domestiques n'évolue pas en fonction de la taille du ménage, la surpopulation favorise la fréquence de vidange des petites fosses et par conséquent elle contribue à l'augmentation des revenus alloués à ce service par le ménage.

La mesure de l'indice de richesse des ménages a permis d'évaluer la capacité à payer des ménages pour le service de vidange (Mbeguere et al., 2011). En effet, une question portant sur le niveau de revenu des ménages rencontrerait une opposition de certains d'entre eux.

L'important pourcentage de chefs de ménage propriétaires de leurs maisons est une situation qui n'est pas contradictoire dans la mesure où il s'agit en générale de la zone périphérique dépourvue de réseaux d'égout.
Néanmoins, même si ce statut n'exclue pas la cohabitation avec d'autres ménages locataires dans la même concession, il garantit de rencontrer des chefs de ménages qui ont une meilleure connaissance de l'historique de la vidange et une maitrise des variables qui impactent son coût.

Le taux d'accès à l'eau potable des ménages de Dakar est le plus élevés du Sénégal. Cette situation est sans doute un préalable à une meilleure gestion de l'assainissement non collectif qui couvre plus de $65 \%$ de la population (Chowdhry et Kone, 2012). En effet avec une population qui pratique majoritairement le lavage anale, l'accès facile à l'eau est une contribution à l'amélioration de l'hygiène.

Tous les types d'ouvrages individuels rencontrés dans les ménages et consignés dans le tableau 6 produisent d'importantes quantités de boues qui requièrent un service de collecte et de transport appropriés (Koné, 2010). Au remplissage d'un ouvrage d'assainissement non collectif, il est nécessaire d'évacuer le contenu pour continuer à l'utiliser (Taweesanet al., 2015). Toutefois, le faible taux de vidangeautomatique observé à Dakar s'explique par le fait que la majeure partie des locataires ne se souviennent pas ou ignorent simplement la fréquence de vidange de leurs ouvrages, puisque, pour les locataires des maisons, c'est le propriétaire qui est responsable de la vidange de la fosse. Cependant, pour $74 \%$ des ménages de Dakar, la vidange est pratiquée au moins une fois par an (Chowdhry et Kone, 2012).

Les ménages utilisent aussi bien la vidange mécanique que la vidange manuelle. Pour plus d'efficacité, il arrive souvent que ces deux modes de vidange soient combinés (Mbéguéréet al., 2010 ; WSA, 2012). Le taux élevé de la vidange manuelle s'explique par plusieurs facteurs dont l'inaccessibilité de certaines fosses par les camions (Walker, 2008), la cherté de la vidange mécanique (Mbeguere et al., 2011), la faible puissance des pompes et la vétusté des camions qui ne parviennent pas à extraire les boues pâteuses 
(Blunier, 2004; Mikhael et al., 2014). Cependant quelle que soit la motivation, le taux élevé de la vidange manuelle peut avoir des conséquences néfastes en raison des nombreux problèmes sanitaires et environnementaux liés à cette activité (Sonko, et al., 2014 ; Seck et al., 2015).

A l'instar de la plupart des villes africaines, l'activité de collecte et de transport des boues de vidange de Dakar ne fait l'objet d'aucune réglementation (Murungi et van Dijk, 2014). Il suffit simplement d'avoir un camion pour se déclarer vidangeur. Ainsi, les ménages desservis par l'assainissement non collectif, pour vidanger leurs fosses se plient aux conditions des opérateurs privés qui fixent les prix sur la base de la couverture de leurs charges et la maximisation de leur profit.

Les prix sont par conséquent jugés inaccessibles par $62 \%$ des ménages. En effet, sous l'influence de la taille des ménages dakarois qui peut atteindre 30 personnes, et du niveau élevé de la nappe phréatique, la fréquence de vidange dans la banlieue de Dakar est d'au moins une vidange par an (Mbéguéré et al, 2010 ; Sonko et al., 2014). Ainsi, la volonté à payer moyenne des ménages s'élève au montant de 14270 FCFA alors que le tarif d'équilibre des entreprises de vidange varie entre 19145 CFA (moyennes entreprise) et $20 \quad 840$ FCFA (grandes entreprises). Il est par conséquent utile de rapprocher les positions pour obtenir un prix d'équilibre accessible aux ménages et soutenable pour les entreprises. Etant donné que la volonté à payer des ménages riches est le double de ceux pauvres (1 000 FCFA), il serait intéressant de fixer les prix en fonction des zones et de la catégorie sociale des ménages en plus de facturer le service de vidange de manière échelonnée comme c'est le cas pour les factures d'eau potable et d'électricité.

En plus de la fréquence de vidange, le consentement à payer des chefs de ménages pour la vidange mécanique de leurs ouvrages domestiques est fonction de la disponibilité et de la qualité du service exprimées respectivement par $37 \%$ et $39 \%$ des chefs de ménages. Cette observation confirme les conclusions des travaux de Blunier (2004) sur les déterminants du prix de la vidange.

La disponibilité du service constitue une préoccupation majeure des chefs de ménages habitants certains quartiers spontanés/irréguliers, inondables, éloignés des sites des stations de traitement des boues de vidange ou difficiles d'accès par manque de voies praticables. Dans ces quartiers, même si des chefs de ménages sont prêts à payer un prix plus élevé, les vidangeurs mécaniques ne sont pas prêts à s'y rendre. Assurément le camionneur commence toujours par évaluer le coût du voyage avant de s'engager. Si le quartier est éloigné, il se pose un problème de perte de temps et de consommation d'importantes quantités de carburant. Pour ce qui est des quartiers spontanés il se pose toujours un problème d'accessibilité. Les routes sont généralement impraticables par les camions qui risquent de s'embourber et perdre des heures de travail. Par ailleurs, les fosses sont souvent installées à l'arrière-cour des maisons nécessitant ainsi beaucoup plus de longueur de flexibles et une pompe avec un bon rendement. En ce qui concerne les quartiers inondés/inondables la contrainte principale qu'évitent les vidangeurs est la remontée de la nappe phréatique. Au cas échéant, le client n'est jamais satisfait de la vidange de la fosse régulièrement alimentée par la nappe.

Les chefs de ménages insistent sur la qualité du service (Semiyaga, 2015). Pour $39 \%$ d'entre eux, ce facteur vient en premier lieu. Ce constat est observé par différentes études antérieures (Mbeguere et al., 2011; ONAS, 2015). La qualité de la vidange mécanique est décriée par certains ménages parce que les pompes des camions ne parviennent pas à aspirer tout le contenu des fosses particulièrement si le fond de la fosse est pâteux (Mbéguéré, 2010 ; Murungi et van Dijk, 2014). En effet, la majeure partie des camions sont de secondes mains. En générale, la première mise en service des camions nouvellement importés d'Europe date de plus de 20 ans (Mbeguere et al., 2011). Ceci 
explique pourquoi les ménages continuent de faire appel aux vidangeurs manuels ou à combiner la vidange mécanique et la vidange manuelle pour garantir l'évacuation complète du contenu de leurs ouvrages et de réduire la fréquence de vidange.

$\mathrm{Au}$ niveau des entreprises privées, la variation des prix du service de la vidange est fonction de la consommation de carburant, des dotations aux amortissements des équipements de vidange, de la masse salariale et de la taxe de dépotage. A l'exception de la consommation du carburant et de la taxe de dépotage, toutes les autres charges sont fixes. Autrement dit, même si l'entreprise n'a pas d'activités, elle doit continuer à les honorer. Ainsi, la charge la plus significative influençant le coût de la vidange demeure la consommation de carburant qui absorbe plus $\mathrm{du}$ tiers des charges variables. Cette forte consommation de carburant s'explique sans doute par l'âge des camions. Ainsi, la consommation d'importantes quantités de carburant d'une moyenne de 15 litres pour chaque voyage de moins de $20 \mathrm{~km}$ est une incidence de l'âge et de la vétusté des camions et des pompes. Ce résultat est confirmé par les conclusions de Chowdhry et Kone (2012). C'est pour cette raison que l'Office National de l'Assainissement du Sénégal (ONAS), conscient de l'impact de l'âge des camions qui influe sur le coût de la vidange a mis en place un fonds de garantie en faveur du financement du renouvellement des camions de boues de vidange de Dakar. Ce fonds est opérationnel depuis janvier 2014.

En définitive, les résultats de cette investigation montrent que les facteurs qui influencent le prix de la vidange mécanique des boues sont donc : la qualité et la disponibilité du service, la fréquence de vidange des fosses et la consommation de carburant liée à l'âge des camions et la distance. Toutefois, les bénéfices d'économie d'échelle observés avec les grandes entreprises encourageraient l'accroissement de la taille des entreprises de vidange pour garantir leur viabilité. Ceci aidera à obtenir des prix équitables, accessibles à un plus grand nombre de ménages.

\section{Conclusion}

La majeure partie des ménages pauvres dans les pays en développement n'arrive pas à s'offrir le service de la vidange mécanique. Plusieurs facteurs concourent à maintenir le prix élevé. Au niveau des ménages, ces facteurs sont la fréquence de la vidange, les caractéristiques des ouvrages de stockage la disponibilité et la qualité du service. Au niveau des entreprises privées, la variation des tarifs du service de la vidange est fonction de quatre variables principales qui par ordre d'importance sont: la consommation de carburant (distance et état du camion), l'amortissement des équipements de vidange, la masse salariale et la taxe de dépotage. La prise en compte de toutes ses charges de fonctionnement révèle que toute entreprise qui pratique uniquement la vidange des boues domestique sans diversification de son activité ne serait pas rentable. Ainsi, dans la logique de minimisation des charges, l'essentielle des entreprises de vidange opèrent dans l'informel. Par ailleurs, la diversification des activités des entreprises de vidange par l'intervention dans le réseau collectif, le nettoiement et le d'autres activités connexes semble être la solution à l'amélioration des revenus des sociétés de vidange. Le renouvellement du parc automobile des vidangeurs est devenue impératif non seulement pour la pérennisation du service de vidange et pour une baisse des tarifs. Toutefois, ce renouvellement n'est possible que par la mise en place de mesures incitatives facilitant l'importation de camions moins âgés. La détaxation des camions importés, l'implantation d'usines de montage de camions de vidange ou la mise sur place de fonds de garanti au profit des opérateurs de la vidange peut participer à cet objectif.

\section{CONFLIT D'INTERETS}

Les auteurs déclarent n'avoir aucun conflit d'intérêts. 


\section{CONTRIBUTIONS DES AUTEURS}

La conception et la planification de l'étude ont été faites par JBG, CD et KD ; l'acquisition des données, l'analyse et l'interprétation par JBG; la rédaction du manuscrit par JBG, $\mathrm{CD}$ et $\mathrm{KD}$; la révision critique par $\mathrm{CD}, \mathrm{KD}$ et $\mathrm{DK}$.

\section{REMERCIEMENTS}

Les auteurs tiennent à remercier l'Institut fédéral suisse des sciences aquatiques et de la technologie (Eawag), le Département de l'assainissement, de l'eau et des déchets solides pour le développement (Sandec), le Centre national de compétence Suisse de Recherche en partenariat Nord-Sud (NCCR North-South), la Fondation nationale suisse pour la science (FNS), la Direction du développement et de la coopération (DDC), la Fondation Bill et Melinda Gates et toutes les institutions participantes.

\section{REFERENCES}

Agassounon-Djikpo-Tchibozo M, Ayi-Fanou L, Lozes E, Fadonougbo R, Anago GDJ, Agbangla C, Ahanhanzo C. 2012. Impacts sanitaires liés à l'usage des eaux de puits, à l'assainissement et à l'aménagement à Gbôdjê dans l'arrondissement de Godomey au Bénin. Int. J. Biol. Chem. Sci., 6(2): 592-602. DOI:

http://dx.doi.org/10.4314/ijbcs.v6i2.4

Akiyo OLR, Afouda AS, YABI I, Adounkpe J, N'bessa B. 2013. Impacts socioéconomiques et environnementaux de la promotion des ouvrages ECOSAN dans le développement de la commune de Sèmè-Podji au sud du Bénin. Int. J. Biol. Chem. Sci., 7(6): 2214-2232. DOI : http://dx.doi.org/10.4314/ijbcs.v7i6.4

ANSD (Agence Nationale de Statistique et de la Démographie). 2014. Recensement Général de la Population et de l'Habitat, de l'Agriculture et de l'Elevage. 2013. Agence Nationale de la Statistique et de la Démographie : Dakar, p.19.

Batsch L. 2003. Rentabilité économique, linéarité de l'investissement et «retour sur dépenses cumulées ", quelques problèmes de mesure. CEREG : Université Paris- Dauphine, p.32.

Blackett I, Hawkins P, Heymans C. 2014. The missing link in sanitation service delivery: A review of fecal sludge management in 12 cities. Water and Sanitation Program. World Bank, p.8.

Blunier P. 2004. La collecte et le transport mécanisés des boues de vidange dans la ville de Ouahigouya (Burkina Faso): Analyse du marché et propositions de réorganisation des flux financiers. Mémoire de master, Ecole Polytechnique Fédérale de Lausanne, Lausanne, p.63.

Chowdhry S, Kone D. 2012. Business Analysis of Fecal Sludge Management: Emptying and Transportation Services in Africa and Asia, Bill \& Melinda Gates Foundation: Seattle USA, P.116.

CSE (Centre de Suivi Ecologique). 2013. Annuaire sur l'environnement et les ressources naturelles du Sénégal (3ième edn). Centre de Suivi Ecologique: Dakar, p.388.

Diene S, Semiyaga S, Niwagaba CB, Muspratt AM, Gning JB, Mbéguéré M, Ennin JE, Zurbrugg C, Strande L. 2013. A value proposition: Resource recovery from faecal sludge-Can it be the driver for improved sanitation? Resources, Conservation and Recycling, 88: 32-38. DOI:

http://dx.doi.org/10.1016/j.resconrec.201 4.04.005

Diop C, Diarra MD, Sonko EHM, Tine M, Matty F, Silva ADS, Dione IPM, Tine A. 2014. Experimental study of slow sand filtration for the treatment of various wastewaters in tropical environment. Int. J. Biol. Chem. Sci., 8(6): 2828-2841. DOI: http://dx.doi.org/10.4314/ijbcs.v8i6.39

Dodane PH, Mbéguéré M, Sow O, Strande L. 2012. Capital and operating costs of fullscale fecal sludge management and wastewater treatment systems in Dakar, Senegal. Environ. Sci. Technol, 46(7) : 3705-3711. DOI: 10.1021/es2045234 
EDE/ICEA. 2009. Etude de la volonté de payer les services d'eau potable et d'assainissement et prévision de la demande en eau potable et en services d'assainissement sur le périmètre de l'hydraulique urbaine. Ministère de l'Urbanisme, de l'Habitat, de l'Hydraulique et de l'Assainissement: Dakar, p.88.

Filmer D, Pritchett LH, 2001. Estimating Wealth Effect without Expenditure Data or Tears: An Application to Educational Enrollments in States of India. Demography, 38(1): 115-132. DOI: 10.1353/dem.2001.0003

Gning JB. 2009. Évaluation socioéconomique de la filière des boues de vidange à Dakar. Mémoire de Diplôme d'Etude Approfondit, Institut des Sciences de l'Environnement, Université Cheikh Anta Diop, Dakar, p.116.

Koné D, Strauss M, Saywell D. 2007 .Vers une Gestion Améliorée des Boues de Vidange (GBV). Rapport du 1er Symposium/Atelier International sur la Politique de Gestion des Boues de Vidange. Eawag : Dübendorf, p.32.

Koné D. 2010. Making urban excreta and wastewater management contribute to cities'economic development: A paradigm shift. Water Policy, 12(4): 602610. DOI: $10.2166 /$ wp. 2010.122

Mbéguéré M, Gning JB, Dodane PH, Koné D. 2010.Socio-economic profile and profitability of faecal sludge emptying companies. Resources, Conservation and Recycling, 54(12): 1288-1295. Doi: 10.1016/j.resconrec.2010.04.008

Mbeguere M, Gning JB, Toure F, Diop B, Toure S, Fam A. 2011. Landscape analysis \& business model assessment in fecal sludge management: extraction and transportation models in Africa-Senegal Bill \& Melinda Gates Foundation. Seattle, p.79.

Mikhael G, Robbins DM, Ramsay JE, Mbéguéré M. 2014. Methods and means for collection and transport of faecal sludge. In IWA Publishing: Faecal
Sludge Management Systems Approach Implementation and Operation, Strande L, Ronteltap M, Brdjanovic D (eds). IWA Publishing: London, p.429.

Murungi c, van Dijk MP. 2014. Emptying, Transportation and Disposal of feacal sludge in informal settlements of Kampala Uganda: The economics of sanitation. Habitat International, 42: 6975.

DOI:

10.1016/j.habitatint.2013.10.011

Ngoutane-Pare MM, Dongo K, Kengne KI, Dodane PH, Akoa A, Kone D. 2012. The economic potential of Echinochloa pyramidalis (Lam.) Hitche \& Chase forage plant used in liquid waste treatment in Cameroon: opportunity to link sanitation to food security. Int. J. Biol. Chem. Sci., 6(1): 210-236. DOI : http://dx.doi.org/10.4314/ijbcs.v6i1.19

ONAS (Office National de l'Assainissement du Sénégal). 2015. Fonds de Garantie en Faveur du Financement des Camions de Boues de Vidange au Sénégal. Programme de Structuration du Marché des Boues de Vidange : Dakar, p.31.

ONAS (Office National de l'Assainissement du Sénégal). 2014. Centre d'appel des boues de vidange: L'outil de l'innovation $\left(\mathrm{N}^{\circ} 2\right)$. Boues mag programme boues de vidange: DakarSénégal. 31.

Pilon M, Vignikin K. 2007. Ménages et familles en Afrique subsaharienne. Éditions des Archives ContemporainesSavoirs Francophones AUF: Paris, p.122.

PSMBV (Programme de Structuration du Marché des Boues de Vidange). 2012. Analyse situationnelle du Programme de Structuration du Marché des Boues de Vidange au bénéfice des populations démunies de la zone périurbaine de Dakar (Pikine et Guédiawaye), Dakar, p.93.

Seck A, Gold, Niang S, Mbéguéré M, Diop C, Strande L. 2015. Faecal sludge drying beds: increasing drying rates for fuel Resource recovery in Sub-Saharan 
Africa. Journal of Water, Sanitation and Hygiene for Development, 5(1): 72-80. Doi: 10.2166/washdev.2014.213

Semiyaga S, Okure MAE, Niwagaba CB, Katukiza AY, Kansiime F. 2015. Decentralized options for faecal sludge management in urban slum areas of SubSaharan Africa: $A$ review of technologies, practices and end-uses. Resources, Conservation and Recycling, 104: 109-119. DOI: 10.1016/j.resconrec.2015.09.001

Sonko, EHM, Mbéguére M, Diop C, Niang S, Strande L. 2014. Effect of hydraulic loading frequency on performance of planted drying beds for the treatment of faecal sludge. Journal of Water, Sanitation and Hygiene for Development, 4(4): 633-641. DOI: 10.2166/washdev.2014.024

SPSS. 2006. SPSS for windows 15.0. SPSS Inc., USA.

Still, D A. 2002. After the pit latrine is full. What then? Effective options for pit latrine management. Biennial Conference of the Water Institute of Southern Africa (WISA) (p. 1). Water Research Commission (WRC): Durban, South Africa.
SUWASA/USAID (Sustainable Water and Sanitation in Africa/United States Agency for International Development). 2014. Rapport d'études du secteur de la gestion des boues de vidange dans la commune de Tambacounda. United States Agency for International Development, Washington DC, p.88.

Taweesan A, Koottatep T, Polprasert C. 2015. Effective faecal sludge management measures for on-site sanitation systems. Journal of Water, Sanitation and Hygiene for Development, 5(3): 483492; DOI: 10.2166/washdev.2015.010

Walker M. 2008. Performance of the FSTP Rufisque and its impact on the WSP. Internship report, ETH - Eawag - ONAS., Dakar p.42.

WMO (World Meteorological Organization) http: //worldweather.wmo.int. (Accessed 22 july 2016).

WSA (Water and Sanitation for Africa). 2012. Benchmarking of Urban Sanitation Pricing and Tariff Structure in Africa. Consolidated Report (Abridged). Water and Sanitation for Africa, Pan-African Intergovernmental Agency: Ouagadougou Burkina Faso, p.24. 